\title{
How Does the pH of a Solvent Influence the Solubility of Turmeric in it?
}

\author{
Miyu Bansal ${ }^{1}$ and Uma Sivakumar ${ }^{1}$
}

${ }^{1}$ Glenunga International High School, Adelaide, South Australia, Australia

\section{ABSTRACT}

Turmeric is a culinary spice used in many meals particularly in traditional Indian cuisine. Turmeric has a bright orange colour that is due to the presence of curcumin within it. Because of it's bright colour, it is important to be able to easily remove turmeric stains from clothing. In my research I attempted to find the ideal conditions for removing turmeric stains and found that no investigation had been made into the impact the $\mathrm{pH}$ of the cleaning agent on its effectiveness.In this investigation the solubility of turmeric in 5 different solutions was found using colorimetry. Colorimetry is a scientific technique that is used to determine the concentration of coloured solutions by the application of the BeerLambert law. This method allowed the solubility of turmeric to be determined, in a range of different solvents with good accuracy and precision. The experimental results showed that turmeric was more soluble in solutions with higher $\mathrm{pH}$ levels as the solubility in $0.2 \mathrm{M}$ sodium hydroxide was $17.6 \mathrm{mg} / \mathrm{mL}$ and in pure ethanol was $10.1 \mathrm{mg} / \mathrm{mL}$, while the solubility in hydrochloric acid was only $0.034 \mathrm{mg} / \mathrm{mL}$. Ultimately, this investigation shows that turmeric is more soluble in basic solutions and hence basic solvents are more effective removers of turmeric stains. A claim which was shown to be true by washing turmeric-stained clothes with the solvents and comparing the cleaned clothes. This investigation also shows that turmeric could be used as a $\mathrm{pH}$ indicator as it has a different colour in different $\mathrm{pH}$ solutions.

\section{Introduction}

Turmeric is a culinary spice that has been used in India for over 4000 years (Ncbi, 2011). I have Indian heritage and as a result, turmeric is an ingredient in many of my day-to-day meals. Once, I was trying to remove a turmeric stain from a white shirt and I saw that when I rubbed it with soap, the stain changed colour from yellow to red. I wanted to understand why this happened and after some investigation into this phenomenon, I decided to do an investigation into the use of turmeric as a $\mathrm{pH}$ indicator and its solubility in solvents of different $\mathrm{pH}$. This led to my research question: "What is the relationship between the $\mathrm{pH}$ of the solvent and the solubility of turmeric?"

I chose to investigate the solubility of turmeric in different $\mathrm{pH}$ solvents, as the solubility of a substance plays an important role in the process of washing clothes, higher solubility leads to better washing power.

In order to approach this research question, I will be designing an experiment to determine the solubility of turmeric in a range of solvents. The solubility of a substance will be determined using colorimetry. Colorimetry is a scientific technique that is used to determine the concentration of coloured solutions by the application of the BeerLambert law (further details in the "Background Theory" section) (Science Direct, 2014). This method will allow the solubility of turmeric to be determined, in a range of different solvents with good accuracy and precision.

There were limited quantities of chemicals available, and limited time. Consequently, the scale of the experiment was reduced, with only five solvents being tested. This was done to ensure that enough repetitions could be performed while also collecting sufficient data to make claims based on the data. 


\section{Background Theory}

Colour changes due to changes in $\mathrm{pH}$ are not uncommon in chemistry, and these changes are often used to determine the $\mathrm{pH}$ of solution (pH Indicators). For instance, litmus paper, figure 1, is used to test whether a solution is acidic or basic. Litmus paper is treated with many halochromic chemical pigments and when it is exposed to acid, the $\mathrm{H}^{+}$ions react with the pigments and the chemical change causes the pigments to reflect a longer wavelength of light resulting in a red colour (Wikipedia, 2020). Generally, pH indicators are weak acids or weak bases that work based on the principal that a change in $\mathrm{pH}$ results in change in ionisation and that the ionized form is of different colour.

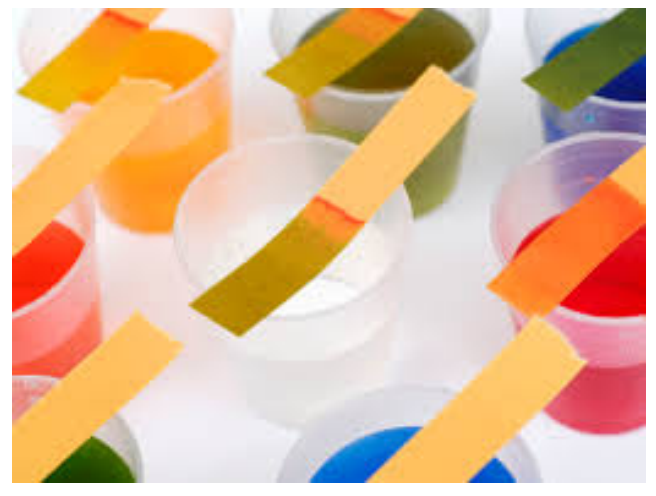

Figure 1. Litmus paper

Turmeric is made of more than 100 different components, but its yellow colour is primarily due to the presence of curcumin within it (Ncbi, 2011). Curcumin is a weakly acidic hydrophobic compound. When curcumin is exposed to weakly basic conditions, its chemical structure experience a subtle change from a diketone form to a keto-enol form, see figure 2. This change causes the compound to change colour from yellow to brown. Moreover, when exposed to strongly basic conditions the molecule ionises, changing from a brown to a reddish-brown colour (Priyadarsini, 2014).

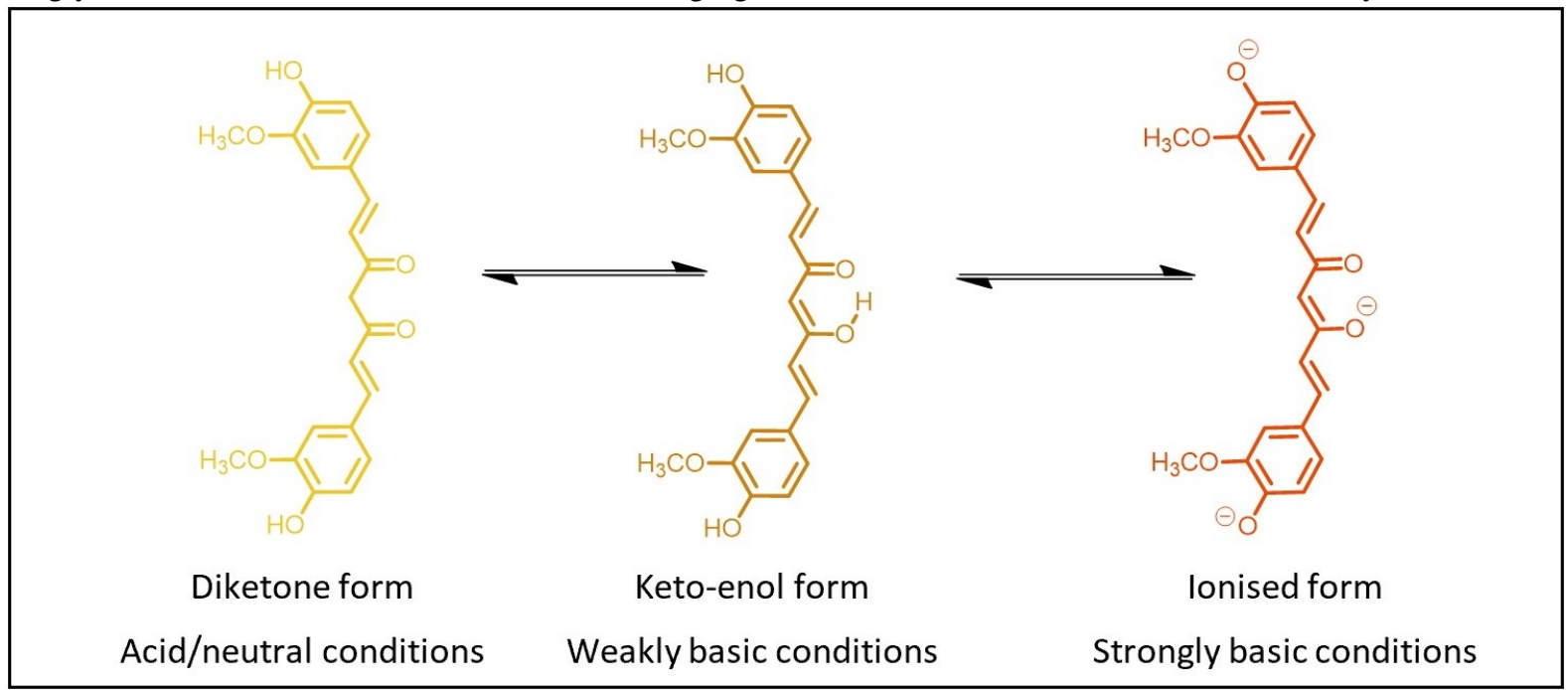

Figure 2. Various forms and colours of curcumin when exposed to acidic or basic conditions

The solubility of a compound, in water, depends upon its polarity and degree of ionisation. For a weak acid, such as curcumin, its $p K_{a}$ value will determine its degree of ionisation, as described by the Henderson-Hasselbalch equation, Equation 1 (Brown and Ford, 2014).

\section{Equation 1:}

$$
p H-p K a=\log \left(\frac{\left[A^{-}\right]}{[H A]}\right)
$$


where $p K_{a}$ is the negative $\log$ of the acid disassociation constant $\left(K_{a}\right),\left[A^{-}\right]$is the concentration of the conjugate base, and $[H A]$ is the concentration of the acid. Using Equation 1, the dependence of the degree of ionisation on $p H$ and $p K a$ is tabulated in the following table, table 1 .

Table 1. Dependence of degree of ionisation on $\mathrm{pH}$ and $\mathrm{pKa}$

\begin{tabular}{|c|c|c|c|}
\hline Condition & $1^{\circ}$ Implication & $2^{\circ}$ Implication & Result \\
\hline$p H=p K a$ & $\log \left\{\left[A^{-}\right] /[H A]\right\}=0$ & {$\left[A^{-}\right]=[H A]$} & Degree of ionisation $=50 \%$ \\
\hline$p H>p K a$ & $\log \left\{\left[A^{-}\right] /[H A]\right\}>1$ & {$\left[A^{-}\right]>[H A]$} & Degree of ionisation $>50 \%$ \\
\hline$p H<p K a$ & $\log \left\{\left[A^{-}\right] /[H A]\right\}<1$ & {$\left[A^{-}\right]<[H A]$} & Degree of ionisation $<50 \%$ \\
\hline
\end{tabular}

As shown in figure 2, keto-enol form of curcumin has 3 acidic protons, and their $p K_{a}$ values are 7.8, 8.5, and 9.0. (ncbi, 2010). As a result, curcumin will be ionised in a solvent of $\mathrm{pH}>7.8$, and the higher the $\mathrm{pH}$ of the solvent, the higher will be its degree of ionisation. Since the solubility a compound, in water, is directly related to its degree of ionisation, the solubility of curcumin and turmeric should be higher in solvents with higher $\mathrm{pH}$.

As previously mentioned, the solubility of turmeric in the different solvents will be determined using colorimetry and the Lambert-Beer law.

Equation 2: The Lambert-Beer law (Brown and Ford, 2014)

$$
A=\varepsilon l c ;
$$

where $A$ is the absorbance, which is the log of the ratio of the intensity of light before and after passing through the sample, $\varepsilon$ is the molar absorptivity, $l$ is the path length and $c$ is the concentration of the solution. The molar absorptivity $(\varepsilon)$ is the absorbance of $1 \mathrm{M}$ solution measured in a cuvette of $1 \mathrm{~cm}$ path length and its unit is $\mathrm{Lmol}^{-1} \mathrm{~cm}^{-1}$. Since the molar absorptivity $(\varepsilon)$ of a substance in a particular solvent is constant and the pathlength $(l)$ is also constant, the absorbance $(A)$ will be directly proportional to the concentration $(c)$. In this experiment, the absorbance of known concentrations of turmeric solutions will be measured and plotted against concentration, producing calibration curves. These calibration curves will then be used to determine the concentration of turmeric in saturated solutions, giving the solubility of turmeric in various solvents. Since $\varepsilon$ is only constant for a particular solvent, 5 calibration curves will be prepared, see figure 4 , as 5 different solvents will be used.

\section{Hypothesis}

Since ionisation of hydrophobic compounds increases their solubility in water and curcumin is a major component of turmeric, the solubility of turmeric should increase as the $\mathrm{pH}$ of the solvent increases and vice versa. Moreover, the turmeric solutions with $p H>7$ should have a red or brown hue.

\section{The Experiment}

\section{Variables}

Independent variable: $\mathrm{pH}$ of solvent

Dependent variables: colour of turmeric solution, absorbance of turmeric solution (used to calculate solubility)

\begin{tabular}{|c|c|}
\hline Controlled Variables & How it will be controlled \\
\hline $\begin{array}{c}\text { Wavelength of light } \\
\text { being passed through } \\
\text { sample }\end{array}$ & $\begin{array}{c}\text { When using the colorimeter to measure the absorbance, the wavelength will always be } \\
\text { set to 430nm. The wavelength will be set at 430nm, as literature shows that in the visi- } \\
\text { ble spectrum, turmeric has maximum absorbance at a wavelength of about 420nm (Jag- } \\
\text { annathan, Abraham and Poddar, 2012). }\end{array}$ \\
\hline
\end{tabular}




\begin{tabular}{|c|c|}
\hline \multicolumn{1}{|c|}{ Path length } & $\begin{array}{c}\text { To ensure that the colorimeter's light beam travels through the same length of sample } \\
\text { in each trial, identical cuvettes of width 10mm will be used throughout the experiment. }\end{array}$ \\
\hline $\begin{array}{c}\text { Uncontrolled variables } \\
\text { concentration within } \\
\text { sample }\end{array}$ & $\begin{array}{c}\text { Incomplete mixing of turmeric solution in cuvette may cause variation in absorbance } \\
\text { measurement, so the samples will be mixed thoroughly using a dropper and multiple } \\
\text { repetitions will be performed. }\end{array}$ \\
\hline $\begin{array}{c}\text { Temperature of sample } \\
\text { Changes in temperature may cause minute changes in the solubility. To reduce changes } \\
\text { in temperature, solutions and mixtures will be kept away from sources of heat, and tri- } \\
\text { als will be conducted in a closed school laboratory. }\end{array}$ \\
\hline
\end{tabular}

\section{Materials}

\begin{tabular}{|c|c|c|}
\hline Glassware and other equipment & Quantity & Precision \\
\hline ISOLAB $25 \mathrm{~mL}$ volumetric flask & 2 & $\pm 0.04 \mathrm{~mL}$ \\
\hline ISOLAB $200 \mathrm{~mL}$ volumetric flask & 5 & $\pm 0.150 \mathrm{~mL}$ \\
\hline 250mL measuring cylinder & 1 & $\pm 1 \mathrm{~mL}$ \\
\hline $25 \mathrm{~mL}$ measuring cylinder & 1 & $\pm 0.25 \mathrm{~mL}$ \\
\hline Ohaus Pioneer Analytical Balance & 1 & $\pm 1 \mathrm{mg}$ \\
\hline Eppendorf adjustable micropipette with tips (range: $2-20 \mu \mathrm{L}$ ) & 1 & $\pm 0.0001 \mathrm{~mL}$ \\
\hline Rainin adjustable pipette with tips (range: $0.5-3 \mathrm{~mL}$ ) & 1 & $\pm 0.0025 \mathrm{~mL}$ \\
\hline Watch glass & 1 & N/A \\
\hline $20 \mathrm{~mL}$ glass vial with screw on lid & 20 & N/A \\
\hline Test tube & 3 & N/A \\
\hline Glass dropper (to mix solution) & 1 & N/A \\
\hline Lab Glass $100 \mathrm{~mL}$ beaker & 5 & N/A \\
\hline Camera (Samsung A70) & 1 & N/A \\
\hline Turmeric stained cloth $(10 \times 15 \mathrm{~cm})$ & 5 & N/A \\
\hline Metal tongs & 1 & N/A \\
\hline Drying rack (to dry wet cloths) & 1 & N/A \\
\hline Plastic funnel & 4 & N/A \\
\hline Spatula & 1 & N/A \\
\hline Filter paper disk & 16 & N/A \\
\hline Plastic disposable cuvettes $(3.5 \mathrm{~mL}, 10 \mathrm{~mm})$ & 42 & N/A \\
\hline Vernier colorimeter & 1 & $\mathrm{~N} / \mathrm{A}$ \\
\hline Data logger (HP Laptop) & 1 & N/A \\
\hline Chemical & Quantity & Concentration \\
\hline Propan-2-ol & $25 \mathrm{~mL}$ & Pure \\
\hline Organic turmeric powder & $20.5 \mathrm{~g}$ & N/A \\
\hline Hydrochloric acid $(\mathrm{HCl})$ solution & $40 \mathrm{~mL}$ & $1 \mathrm{M}$ \\
\hline Sodium hydroxide $(\mathrm{NaOH})$ powder & $1.6 \mathrm{~g}$ & $\mathrm{~N} / \mathrm{A}$ \\
\hline Ethanol & $300 \mathrm{~mL}$ & Pure \\
\hline Distilled water & $660 \mathrm{~mL}$ & $\mathrm{~N} / \mathrm{A}$ \\
\hline
\end{tabular}




\section{Experimental procedure}

\section{A) Preparing stock solutions}

Turmeric stock solution

1. Add approx. $5 \mathrm{~mL}$ of propan-2-ol to the $25 \mathrm{~mL}$ volumetric flask

2. Using the digital scale weigh $200 \mathrm{mg}$ of turmeric powder on a watch glass

3. Use the plastic funnel to add the $200 \mathrm{mg}$ of turmeric powder to the volumetric flask containing the propan2-ol

4. Use propan-2-ol to wash the watch glass and funnel, ensuring that all turmeric goes into the volumetric flask, and that the volume does not exceed $25 \mathrm{~mL}$

5. Add propan-2-ol until bottom of meniscus is level with $25 \mathrm{~mL}$ mark

6. Close the flask and shake vigorously

7. Heat solution to about $50^{\circ} \mathrm{C}$ and shake again

8. After solution has cooled, fold a filter paper disk into a cone and use the filter paper cone and a plastic funnel to filter the solution into a clean $25 \mathrm{~mL}$ volumetric flask

9. After solution has finished filtering, allow filter paper to dry and weigh filter paper with the excess turmeric

$\mathrm{pH}$ stock solutions

1. Add $200 \mathrm{~mL}$ of distilled water to a $200 \mathrm{~mL}$ volumetric flask (distilled water stock)

2. Add $100 \mathrm{~mL}$ of distilled water and $100 \mathrm{~mL}$ of ethanol to a $200 \mathrm{~mL}$ volumetric flask using a $250 \mathrm{~mL}$ measuring cylinder (50\% ethanol stock)

3. Add $200 \mathrm{~mL}$ of ethanol to a $200 \mathrm{~mL}$ volumetric flask (ethanol stock)

4. Add $1.6 \mathrm{~g}$ of $\mathrm{NaOH}$ and $200 \mathrm{~mL}$ of distilled water to a $200 \mathrm{~mL}$ volumetric flask $(0.2 \mathrm{M} \mathrm{NaOH}$ stock)

5. Add $40 \mathrm{~mL}$ of $1 \mathrm{M} \mathrm{HCl}$ solution to a $200 \mathrm{~mL}$ volumetric flask and fill with distilled water to mark $(0.2 \mathrm{M} \mathrm{HCl}$ stock)

\section{B) Determining the colour change}

1. Add $15 \mathrm{~mL}$ of each $\mathrm{pH}$ stock solution to 5 glass vials

2. Add approx. $5 \mathrm{mg}$ of turmeric powder to each vial

3. Close the vials and shake vigorously

4. Observe the colour change and take pictures and record observations as required

\section{C) Preparing the calibration curves}

1. Setup the colorimeter by connecting it to the data logger and set the wavelength to $430 \mathrm{~nm}$

2. Add $3 \mathrm{~mL}$ of the $\mathrm{HCl}$ stock to a cuvette

3. Place the cuvette in the colorimeter and press the calibrate button

4. After the colorimeter has been calibrated (absorbance $=0$ ), using the micropipette add $10 \mu \mathrm{L}$ of the turmeric stock solution to the cuvette and thoroughly mix the cuvette with the glass dropper

5. Once the absorbance measurement is stable, record the absorbance

6. Repeat steps $4-5$ four times

7. Repeat steps $2-6$ for each $\mathrm{pH}$ stock solution 


\section{D) Determining the solubility of turmeric in each $\mathrm{pH}$ stock solution}

1. Take three $25 \mathrm{~mL}$ vials and add $15 \mathrm{~mL}$ of the $\mathrm{HCl}$ stock solution to each vial using a $25 \mathrm{~mL}$ measuring cylinder

2. Add excess turmeric powder (approximately $1.0 \mathrm{~g}$ ) and close the vial

3. Shake the mixture vigorously for 5 minutes

4. Use the adjustable pipette to add $3 \mathrm{~mL}$ of $\mathrm{HCl}$ stock into a cuvette, place the cuvette in the colorimeter and press the calibrate button

5. Fold a filter paper disk into a cone, and use the cone and a plastic funnel to filter the mixture from one vial into a test tube, see figure 3 .

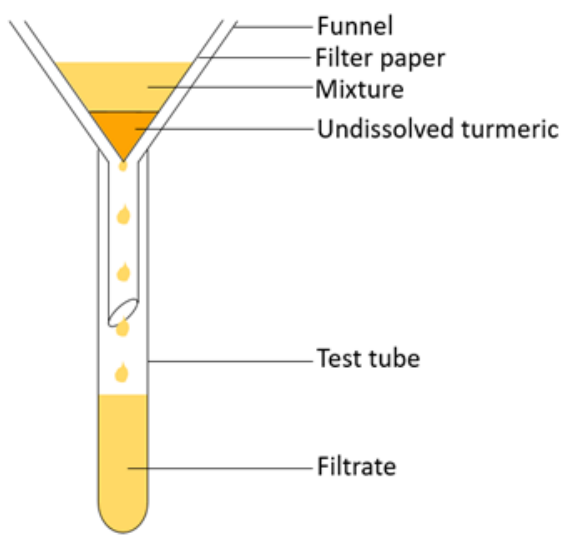

Figure 3. Filtration setup

6. Using the adjustable pipette, add $3 \mathrm{~mL}$ of the filtrate from one test tube into a cuvette and observe the absorbance. If the absorbance is outside the range of the calibration curves the filtrates will need to be diluted (the $\mathrm{HCl} \&$ water filtrates were not diluted, to dilute the ethanol and $50 \%$ ethanol filtrates $10 \mu \mathrm{L}$ of the filtrate was added to $2 \mathrm{~mL}$ of the respective stock solution and to dilute the $\mathrm{NaOH}$ filtrate $10 \mu \mathrm{L}$ of the filtrate was added to $3 \mathrm{~mL}$ of the $\mathrm{NaOH}$ stock solution)

7. After diluting the filtrates as required, use the adjustable pipette to add the filtrate from one test tube (set 1) to three cuvettes ( $3 \mathrm{~mL}$ each, creating 3 trials per set)

8. Measure the absorbance for all 3 cuvette and record the values in a table and record any qualitative observations

9. Repeat steps 1-8 twice (sets 2 and 3), resulting 9 observations for $\mathrm{HCl}$ Repeat steps 1-9 for all $\mathrm{pH}$ stock solutions

\section{E) Determining the washing power of the pH stock solvents}

1. Add $50 \mathrm{~mL}$ of the $\mathrm{HCl}$ stock to a $100 \mathrm{~mL}$ beaker

2. Repeat step 1 for all $\mathrm{pH}$ stock solutions

3. Add one turmeric stained cloth to each beaker and mix with metal tongs (make sure entire cloth is submerged within the solution)

4. Allow to soak for 10 minutes

5. Use tongs to remove cloths from beakers and hang cloths on drying rack

6. Once cloths have dried, take pictures of cloths and take pictures of solution remaining in beakers

7. Note down any qualitative observations 


\title{
Risk Assessment
}

\author{
Chemical risks and handling
}

- Goggles, aprons and covered shoes must be worn at all times

- Ethanol is highly flammable and can cause irritation to eyes and skin. Ethanol must be used in a well-ventilated lab, with appropriate equipment for extinguishing fires. Ethanol must be kept away from ignition sources. If ethanol needs to be heated use a spark-proof water bath, never use an open flame (Ethanol, 2019).

- $\mathrm{HCl}(<2.7 \mathrm{M})$ may cause harm in the eyes or in a cut. Wear eye protection and gloves. Avoid breathing and $\mathrm{HCl}$ fumes. (Hydrochloric acid, 2019).

- Propan-2-ol is highly flammable and can cause irritation to the eyes. Propan-2-ol must be used in a wellventilated lab, with appropriate equipment for extinguishing fires. Propan-2-ol must be kept away from ignition sources. If propan-2-ol needs to be heated use a spark-proof water bath, never use an open flame (Higher alcohols, 2019).

- $\mathrm{NaOH}$ can cause severe skin burns and eye damage. Wear eye protection and protective gloves (Sodium hydroxide, 2019).

\section{Procedural risks and disposal}

- Colorimeter light can cause severe eye damage. Do NOT look directly into the colorimeter's intense light source. Before using colorimeter, check that the colorimeter has a valid safety tag.

- Glassware can break or chip. Broken/chipped glassware can cause cuts. Any chipped or cracked glassware should be inspected and discarded. Any broken glass should be swept up with a brush and dustpan; do NOT use fingers.

- Disposal all chemicals in the chemical sink

\section{Emergency treatment}

- Ethanol: If comes in contact with the eye, flood eyes with tap water for 10 minutes. If swallowed wash out mouth with water. Do NOT induce vomiting. If comes in contact with skin/clothing, remove contaminated clothing and thoroughly wash affected area with water (Ethanol, 2019).

- $\mathrm{HCl}(<3 \mathrm{M})$ : If comes in contact with the eye, flood eyes with tap water for 10 minutes. If vapour breathed in, move to fresh air and consult medic if irritation continues. If swallowed, wash out mouth with drinking water. Do NOT induce vomiting. If comes in contact with skin/clothing, remove contaminated clothing and drench skin with plenty of water (Hydrochloric acid, 2019).

- Propan-2-ol: If comes in contact with the eye, flood eyes with tap water for 10 minutes. If vapour breathed in, move to fresh air and consult medic if breathing is difficult. If swallowed, wash out mouth with water. Do NOT induce vomiting. If comes in contact with skin/clothing, remove contaminated clothing and if more than a test tube amount was spilt, wash the affected area with plenty of water (Higher alcohols, 2019).

- NaOH: If comes in contact with the eye, flood eyes with gently-running tap water for at least 20 minutes and consult a medic. If swallowed, wash out mouth with water. Do NOT induce vomiting. If comes in contact with skin/clothing, remove contaminated clothing and drench skin with plenty of water (Sodium hydroxide, 2019).

- Glassware: In the event of an injury involving broken glass, wash the wound thoroughly with warm water and soap. Following this, seek medication attention as required. 
- Colorimeter: In the event of a severe eye injury, caused by looking into the bright light source of the colorimeter, immediately seek appropriate medical attention.

\section{Results}

\section{Preparing the calibration curves}

In accordance with the method outlined in part $\mathrm{C}$ of the experimental procedure section, the following absorbance vs. concentration data was recorded. Sample calculations are presented below the table and the data highlighted red was used for the sample calculations. The concentration vs. absorbance data, from table 2, is plotted on figure 4. Following the Lambert-Beer law, the data was fit into linear function using MS Excel, and the line equations along with the correlation coefficients are presented on figure 4 . The trend lines were constrained so that the y-intercept is zero.

Table 2. Absorbance vs. concentration data for preparation of calibration curves

\begin{tabular}{|c|c|c|c|c|c|c|c|}
\hline \multirow{2}{*}{$\begin{array}{l}\text { Volume of Turmeric } \\
\text { Stock Solution added } \\
(\mathrm{mL})\end{array}$} & \multirow{2}{*}{$\begin{array}{l}\text { Total Volume in } \\
\text { Cuvette (mL) }\end{array}$} & \multirow{2}{*}{$\begin{array}{l}\text { Concentration } \\
(\mathrm{mg} / \mathrm{mL})\end{array}$} & \multicolumn{5}{|c|}{ Absorbance $( \pm 0.001)$} \\
\hline & & & $\mathrm{NaOH}$ & Water & $\mathrm{EtOH}$ & $50 \% \mathrm{EtOH}$ & $\mathrm{HCl}$ \\
\hline $0.0000 \pm 0.0000$ & $3.0000 \pm 0.0025$ & $0.000 \pm 0.000$ & 0.000 & 0.000 & 0.000 & 0.000 & 0.000 \\
\hline $0.0100 \pm 0.0001$ & $3.0100 \pm 0.0026$ & $0.0159 \pm 0.006$ & 0.095 & 0.041 & 0.085 & 0.078 & 0.060 \\
\hline $0.0200 \pm 0.0002$ & $3.0200 \pm 0.0027$ & $0.032 \pm 0.001$ & 0.189 & 0.083 & 0.169 & 0.156 & 0.116 \\
\hline $0.0300 \pm 0.0003$ & $3.0300 \pm 0.0028$ & $0.048 \pm 0.002$ & 0.279 & 0.124 & 0.252 & 0.231 & 0.175 \\
\hline $0.0400 \pm 0.0004$ & $3.0400 \pm 0.0029$ & $0.063 \pm 0.002$ & 0.369 & 0.163 & 0.332 & 0.303 & 0.230 \\
\hline $0.0500 \pm 0.0005$ & $3.0500 \pm 0.0030$ & $0.079 \pm 0.003$ & 0.456 & 0.201 & 0.409 & 0.371 & 0.286 \\
\hline
\end{tabular}

\section{Sample calculation of concentration (data highlighted red):}

Abbreviations:

- $\quad$ s. $f .=$ significant figures

- $\quad$ d.p. $=$ decimal places

Concentration of turmeric in stock solution:

Mass of turmeric dissolved $=$ total mass of turmeric - (mass of used filter paper with excess turmeric - mass of dry filter paper)

mass of turmeric dissolved $=200 \pm 1-((826 \pm 1)-(746 \pm 1))$

$=120 \pm 3 \mathrm{mg}$

Volume of propan-2-ol $=25.00 \pm 0.04 m L$

$$
\begin{aligned}
\text { Concentration } & =\frac{\text { mass of turmeric dissolved }}{\text { volume of isopropanol }} \\
& =\frac{120 \pm 3}{25.00 \pm 0.04} \\
& =4.80 \pm\left(\frac{3}{120} \times 100 \%+\frac{0.04}{25.00} \times 100 \%\right) \\
& =4.80 \pm(2.66 \%) \\
& =4.8 \pm 0.1 \mathrm{mg} / \mathrm{mL}
\end{aligned}
$$

Uncertainty is given to 1 s.f. as the percentage uncertainty is greater than $2 \%$, concentration is given as 4.8 to maintain same d.p. 
Concentration of turmeric in solution in cuvette:

The following equation will be used, $C_{1} \times V_{1}=C_{2} \times V_{2}$

Initial concentration $=4.8 \pm 0.1$

Initial volume $=(0.0100 \pm 0.0001) \times 4$

$$
=0.0400 \pm 0.0004 m L
$$

Final volume $=(3.0000 \pm 0.0025)+(0.0400 \pm 0.0004)$

$$
=3.0400 \pm 0.0029 \mathrm{~mL}
$$

$C_{2}=\frac{C_{1} \times V_{1}}{V_{2}}=\frac{(4.8 \pm 0.1) \times(0.0400 \pm 0.0004)}{3.0400 \pm 0.0029}$

$=\frac{4.8 \times 0.0400}{3.0400} \pm\left(\frac{0.1}{4.8} \times 100 \%+\frac{0.0004}{0.0400} \times 100 \%+\frac{0.0029}{3.0400} \times 100 \%\right)$

$=0.06316 \pm(3.76 \%)$

$=0.063 \pm \frac{0.002 m g}{m L}$

Uncertainty is given to 1 s.f. as the percentage uncertainty is greater than $2 \%$, concentration is given as 0.063 to maintain same d.p.

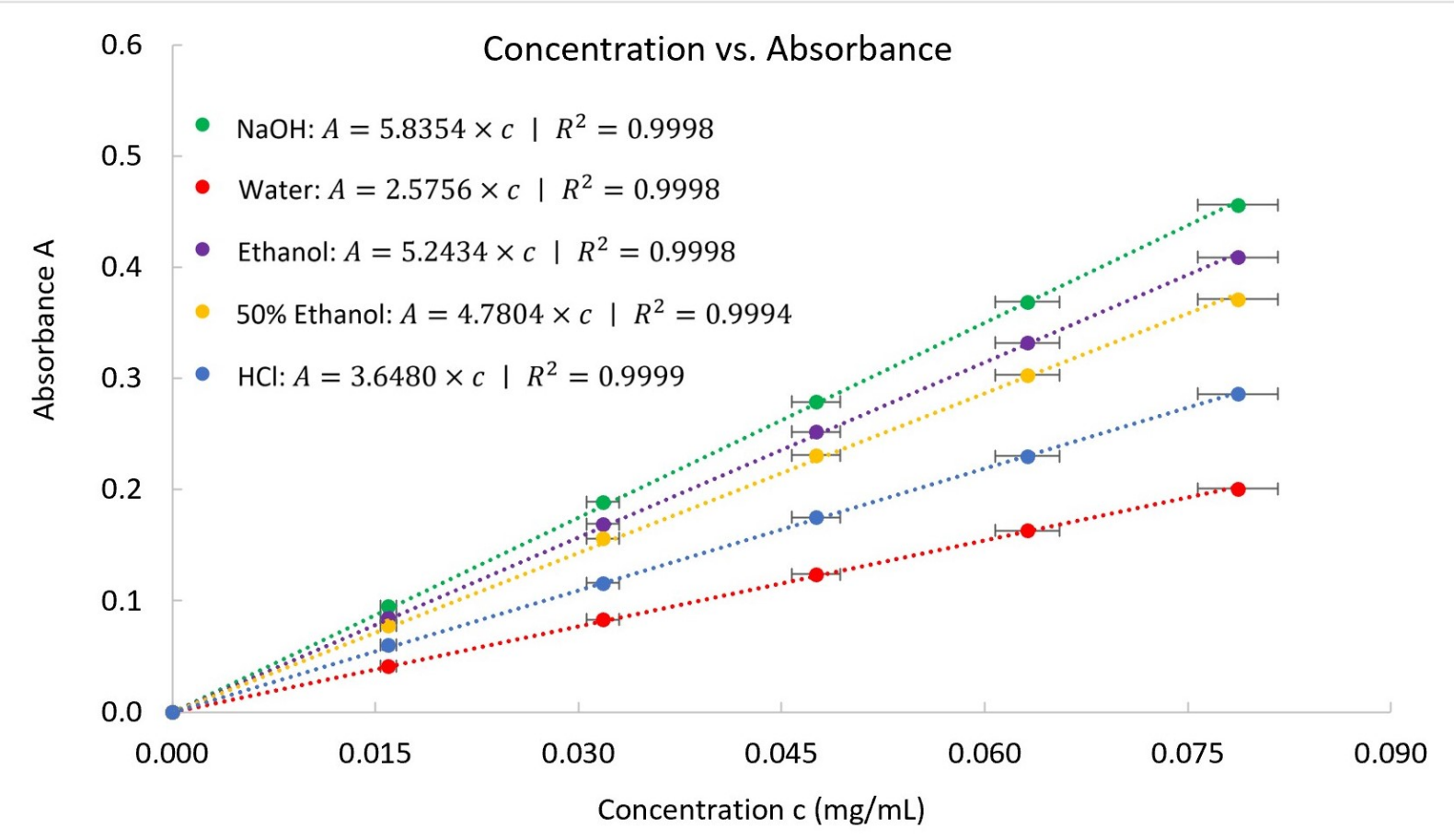

Figure 4. Concentration vs. absorbance scatter plot (calibration curves)

Determining the solubility of turmeric in each $\mathrm{pH}$ solution

Following the method outlined in part D of the experimental procedure section the following absorbance data for the various turmeric filtrates was recorded. For each solvent, there are three sets and each set has three absorbance readings, totalling nine readings per solvent. The data is presented in table 3 .

Table 3. Absorbance of diluted filtrates

\begin{tabular}{|c|c|c|c|}
\hline \multirow{2}{*}{ Solvent } & Absorbance ()$( \pm 0.001)$ & Set 2 & Set 3 \\
\cline { 2 - 4 } & Set 1 & 0.331 & 0.33 \\
\hline
\end{tabular}




\begin{tabular}{|c|c|c|c|}
\hline \multirow{3}{*}{ Ethanol } & 0.335 & 0.352 & 0.347 \\
\cline { 2 - 4 } & 0.351 & 0.342 & 0.329 \\
\hline \multirow{3}{*}{$50 \%$ Ethanol } & 0.26 & 0.271 & 0.265 \\
\cline { 2 - 4 } & 0.255 & 0.268 & 0.259 \\
\hline \multirow{3}{*}{ Water } & 0.267 & 0.263 & 0.27 \\
\hline & 0.173 & 0.18 & 0.191 \\
\hline \multirow{3}{*}{$\mathrm{HCl}$} & 0.166 & 0.176 & 0.186 \\
\hline & 0.171 & 0.178 & 0.183 \\
\hline & 0.193 & 0.192 & 0.183 \\
\hline & 0.169 & 0.186 & 0.168 \\
\hline & 0.175 & 0.179 & 0.12 \\
\hline & 0.14 & 0.11 & 0.125 \\
\hline & 0.136 & 0.113 & 0.115 \\
\hline
\end{tabular}

Qualitative observations:

- $\quad \mathrm{NaOH}$ and ethanol solutions dissolve much more turmeric compared to $\mathrm{HCl}$ and water

- The $\mathrm{NaOH}$ turmeric solution was more reddish in comparison to the other solutions

Using the calibration curves prepared in the previous section, the absorbance measurements were used to calculate the concentration of turmeric in the samples. The concentration values are presented in table 4. Sample calculations of the concentration for the red data are given below the table.

Table 4. Concentration of saturated solution of diluted filtrates

\begin{tabular}{|c|c|c|c|}
\hline \multirow{2}{*}{ Solvent } & \multicolumn{3}{|c|}{ Concentration of turmeric in saturated solution $(\mathrm{mg} / \mathrm{mL})$} \\
\hline & Set 1 & Set 2 & Set 3 \\
\hline \multirow{3}{*}{$\mathrm{NaOH}$} & $17.847 \pm 0.052$ & $17.074 \pm 0.052$ & $17.022 \pm 0.052$ \\
\hline & $17.280 \pm 0.052$ & $18.157 \pm 0.052$ & $17.899 \pm 0.052$ \\
\hline & $18.105 \pm 0.052$ & $17.641 \pm 0.052$ & $16.970 \pm 0.052$ \\
\hline \multirow{3}{*}{ Ethanol } & $9.967 \pm 0.038$ & $10.388 \pm 0.038$ & $10.158 \pm 0.038$ \\
\hline & $9.775 \pm 0.038$ & $10.273 \pm 0.038$ & $9.928 \pm 0.038$ \\
\hline & $10.235 \pm 0.038$ & $10.082 \pm 0.038$ & $10.350 \pm 0.038$ \\
\hline \multirow{3}{*}{$50 \%$ Ethanol } & $7.274 \pm 0.042$ & $7.568 \pm 0.042$ & $8.031 \pm 0.042$ \\
\hline & $6.980 \pm 0.042$ & $7.400 \pm 0.042$ & $7.821 \pm 0.042$ \\
\hline & $7.190 \pm 0.042$ & $7.484 \pm 0.042$ & $7.695 \pm 0.042$ \\
\hline \multirow{3}{*}{ Water } & $0.07493 \pm 0.00039$ & $0.07455 \pm 0.00039$ & $0.07105 \pm 0.00039$ \\
\hline & $0.06562 \pm 0.00039$ & $0.07222 \pm 0.00039$ & $0.07649 \pm 0.00039$ \\
\hline & $0.06795 \pm 0.00039$ & $0.06950 \pm 0.00039$ & $0.06523 \pm 0.00039$ \\
\hline \multirow{3}{*}{$\mathrm{HCl}$} & $0.03838 \pm 0.00027$ & $0.03015 \pm 0.00027$ & $0.03289 \pm 0.00027$ \\
\hline & $0.03728 \pm 0.00027$ & $0.03098 \pm 0.00027$ & $0.03427 \pm 0.00027$ \\
\hline & $0.03646 \pm 0.00027$ & $0.02961 \pm 0.00027$ & $0.03152 \pm 0.00027$ \\
\hline
\end{tabular}

Sample calculation of concentration of turmeric (data highlighted red):

Using the calibration curve equation for $\mathrm{NaOH}$,

$c_{\text {diluted }}=\frac{A}{5.8354}$

$c_{\text {diluted }}=\frac{0.352 \pm 0.001}{5.8354}$ 


$$
\begin{aligned}
& =\frac{0.352}{5.8354} \pm\left(\frac{0.001}{0.352} \times 100 \%\right) \\
& =0.06032 \pm(0.284 \%) \\
& =0.06032 \pm 0.00017 \mathrm{mg} / \mathrm{mL}
\end{aligned}
$$

To calculate the concentration of turmeric in the undiluted solution the following equation will be used, $c_{\text {undiluted }} \times V_{\text {inital }}=c_{\text {diluted }} \times V_{\text {final }}$

$V_{\text {initial }}=10 \mu \mathrm{L}$ and $V_{\text {final }}=10 \mu \mathrm{L}+3 \mathrm{~mL}=3010 \mu \mathrm{L}$

$c_{\text {undiluted }}=(0.06032 \pm 0.00017) \times \frac{3010}{10}$

$c=18.157 \pm 0.052 \mathrm{mg} / \mathrm{mL}$

Uncertainty is given to 2 s.f. as the percentage uncertainty is less than $2 \%$, concentration is given as 18.157 to maintain same d.p.

The concentration data of each solvent was averaged. Since the samples were saturated, the concentration of turmeric is equivalent to the solubility of turmeric in each solvent. The final solubility data is presented in table 5. Sample calculations of the average concentration of turmeric in ethanol, red data, are presented below the table. Table $\mathbf{5}$.

\begin{tabular}{|c|c|c|c|c|c|}
\hline Solvent & $0.2 \mathrm{M} \mathrm{NaOH}$ & Ethanol & $50 \%$ Ethanol & Water & $0.2 \mathrm{M} \mathrm{HCl}$ \\
\hline $\mathrm{pH}$ & 13.30 & 7.33 & 7.13 & 7.00 & 0.70 \\
\hline Solubility of turmeric $(\mathrm{mg} / \mathrm{mL})$ & $17.6 \pm 0.5$ & $10.1 \pm 0.2$ & $7.5 \pm 0.3$ & $0.071 \pm 0.004$ & $0.034 \pm 0.003$ \\
\hline
\end{tabular}

Solubility of turmeric in each solvent with $\mathrm{pH}$ values

\section{Sample calculation of average concentration of turmeric (data highlighted red):}

$$
\begin{aligned}
& \text { Average concentration }=\text { average of all } 9 \text { trials } \\
& \begin{aligned}
\text { Average concentration } \\
=\frac{9.967+9.775+10.235+10.388+10.273+10.82+10.158+9.928+10.350}{9} \\
=\frac{91.158}{9} \\
=10.1 \pm 0.2 \mathrm{mg} / \mathrm{mL}
\end{aligned}
\end{aligned}
$$

Uncertainty was taken to be the standard deviation in the concentration values. Uncertainty is given to 1 s.f. as the percentage uncertainty is greater than $2 \%$, average concentration is given as 10.1 to maintain same d.p.

\section{Determining colour change and determining washing power of each solvent}

According to the method described in part B of the experimental procedure section, a fixed amount of turmeric powder was added to $15 \mathrm{~mL}$ of each solvent and mixed until dissolved (the turmeric did not fully dissolve in the $\mathrm{HCl}$ and water). After the solutions settled, a picture was taken, figure 5. 


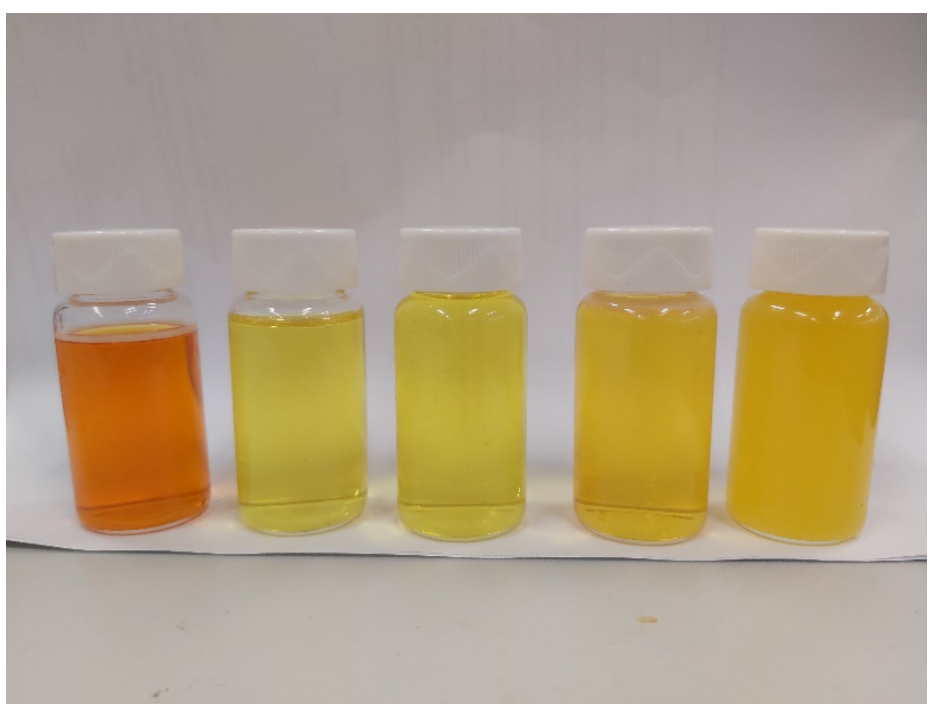

Figure 5. Colour of turmeric in each solvent

Following the method described in part $\mathrm{E}$ of the experimental procedure, five turmeric stained cloths were soaked in the 5 solvents for 10 minutes. After soaking, the cloths were removed from the solvents and allowed to dry. The cloths after washing are shown in figure 6, and the colours of the solvents remaining are shown in figure 7.
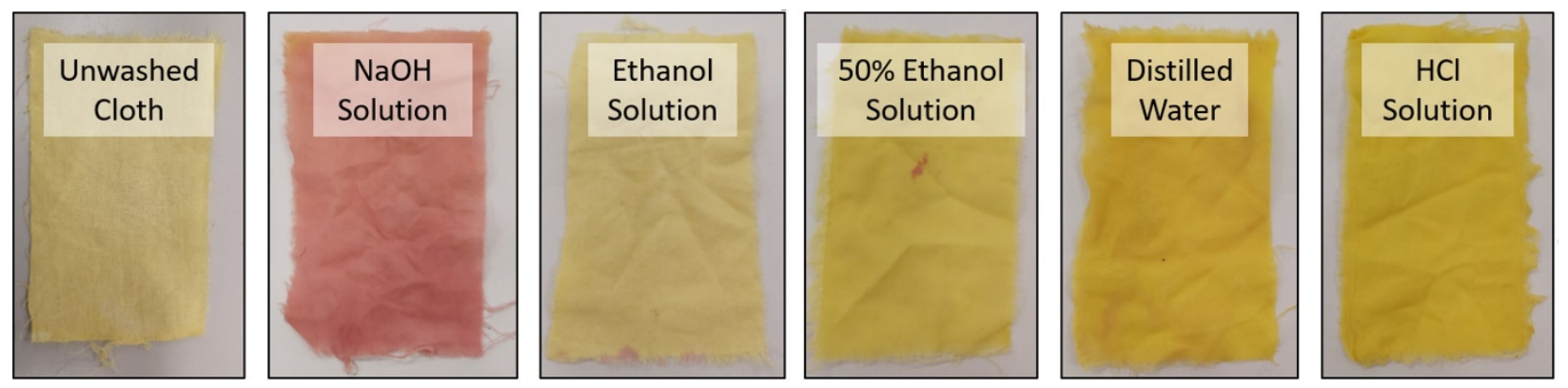

Figure 6. Turmeric stained cloths before and after washing
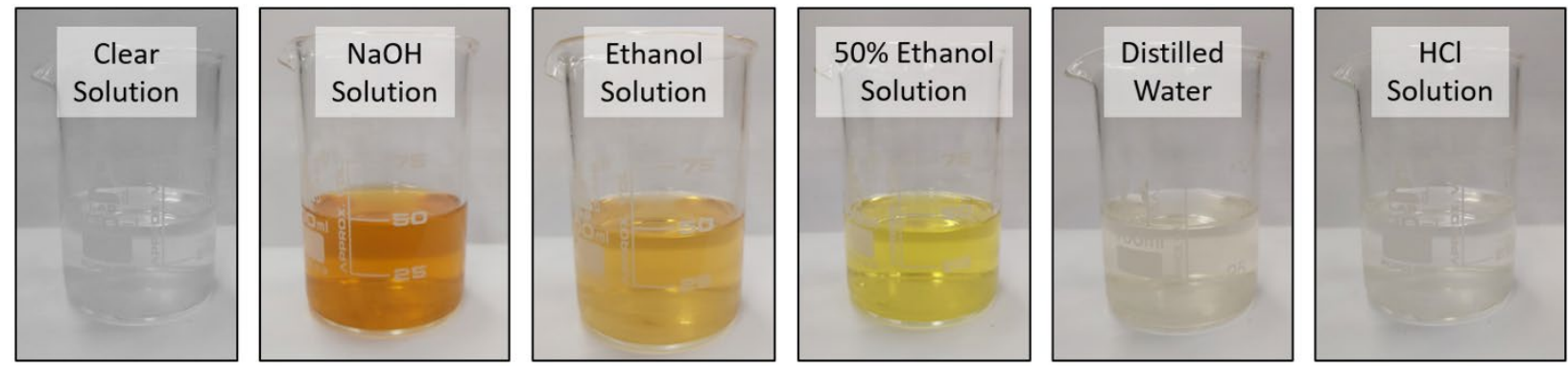

Figure 7. Solvents before and after being used to wash turmeric stained cloths

\section{Interpretation of Results}

As shown in figure 4, the colorimeter produced calibration curves in concentration range of 0.015 to $0.079 \mathrm{mg}$ of turmeric in $1 \mathrm{ml}$ of each solvent. As each calibration curve has high correlation coefficient $\left(r^{2}>0.99\right)$, the calibration curves closely follow a linear trend, indicating the presence of minimal random error. As reported in table 7, turmeric powder has a very high solubility in $0.2 \mathrm{M} \mathrm{NaOH}$ solution $(17.6 \pm 0.5 \mathrm{mg} / \mathrm{mL})$, which is most likely due to ionisation 
of the acidic $\mathrm{OH}$ groups (Priyadarsini, 2014). The brownish-red colour of the turmeric $\mathrm{NaOH}$ solution indicates the formation of phenoxide anion, further supporting the ionisation of the $\mathrm{OH}$ groups. The turmeric was also relatively soluble in ethanol $(10.1 \pm 0.2 \mathrm{mg} / \mathrm{mL})$ and $50 \%$ ethanol $(7.5 \pm 0.3 \mathrm{mg} / \mathrm{mL})$; however, its solubility in water $(0.071 \pm 0.004 \mathrm{mg} / \mathrm{mL})$ and in the $0.2 \mathrm{M} \mathrm{HCl}$ solution $(0.034 \pm 0.003 \mathrm{mg} / \mathrm{mL})$ was very poor. The three $\mathrm{OH}$ groups of curcumin allow it to form hydrogen bonding with ethanol and water, and as a result it should be soluble in both solvents. However the hydrophobic benzene rings dominates and as a result, the solubility in water is limited and the solubility in solvents that are both protic (capable of forming hydrogen bonds) and organic is high, such as ethanol. This observation is also supported by fact that mixture water-ethanol mixture has medium solubility. Similar to water, $\mathrm{HCl}$ does not dissolve curcumin due to its hydrophobic benzene rings. The various solubility values support my hypothesis that solubility of curcumin will be higher in higher $\mathrm{pH}$ solvents. Furthermore, the soaking of identical turmeric stained cloths left lots of colour in the $0.2 \mathrm{M} \mathrm{NaOH}$ solution and ethanol, whereas the yellow colour in the water and $0.2 \mathrm{M} \mathrm{HCl}$ solution was almost undetectable. Evidently, turmeric dissolves more in solvents that are more basic and, as shown by figure 7, basic solutions/soaps are much more efficient removers of turmeric stains. Additionally, since the change in colour due to $\mathrm{pH}$ is quite significant, figure 5, turmeric could be used as an indicator to distinguish between acids and bases.

\section{Conclusion}

The results (table 7 and figure 7) show that turmeric is more soluble in solvents with higher concentrations of hydronium ions $\left(\left[\mathrm{OH}^{-}\right]\right)$and the implication of this is that solvents that are more basic remove turmeric stains more efficiently. Moreover, from figure 5, it is evident that the $\mathrm{pH}$ of the solvent can greatly influence the colour of the turmeric solution, and as a result, turmeric could be used as a $\mathrm{pH}$ indicator.

However, the final solubility values have high level of uncertainty and the experimental method has many weaknesses and strengths, as discussed below.

\section{Strengths and Weaknesses}

In terms of strengths, the use of colorimetry was very effective, as it allowed the solubility of turmeric to be measured quickly and efficiently, permitting the multiple repetitions. Moreover, experiment used to determine the washing power of the different solvents clearly demonstrates the implications of the results and justifies the investigation, as it shows that the results have a real-world application.

In terms of weaknesses, due to time constraints, when preparing the calibration curves, only one repetition was performed for each concentration, which significantly reduced the reliability of the calibration curves. Moreover, the time limitation also meant that only five solvents, rather than seven, were tested, (initially the plan also included testing citric acid and sodium bicarbonate).

\section{Random errors:}

By propagating the uncertainties throughout the calculations, some of the random error has been accounted for. Moreover, the high correlation coefficients of the calibration curves suggest only minimal random error. Despite this, some random errors are inevitable. The following errors may be have been accounted for in the uncertainty or overlooked:

- Throughout the process of measuring the absorbance values for the calibration curves, there was usually some residue in the glass dropper used to mix the samples. This is a random error as the residue remaining would definitely not be constant and as a result, in some samples the volume will be slightly lower, causing loss of precision in the recorded concentrations. This could be reduced through more repetitions, but as 
mentioned previously due to time constraints only one measurement was taken for all of the calibration curve data.

- Throughout the experimental period, many students were performing experiments and due to the limited workspace, some of the samples may have been exposed to sources of heat. This is a random error as temperature can influence solubility and the inconsistent temperatures may result in some samples having a higher or lower solubility. This could be improved by storing the samples in a temperature controlled zone in between trials, but due to the limited workspace, this would likely not be possible.

\section{Systematic errors:}

- When preparing the turmeric stock solution used when preparing the calibration curves, the mass of turmeric that dissolved was taken to be total mass minus the mass remaining on filter paper, but since the paper was not completely dry, some of the weight would have been caused by propan-2-ol residue. As a result, the concentration of the turmeric stock should be slightly higher and hence, all of the turmeric solubility values should be slightly higher. This could be improved by dissolving a fixed amount of turmeric that is less than the theoretical maximum, allowing the concentration value to be more accurate.

\section{Mistakes}

Despite the simplicity and repeatability of the method, throughout the experimental period some mistakes were made.

- In some trials the wrong side of the cuvette was accidentally held, which would result in their being grease on the cuvette. This grease would reduce the absorbance in some trials and thus would decrease the precision. This could be improved by being more careful, or by performing more trials to reduce the impact of this mistake.

- In some trials, the mixture may not have been mixed uniformly and as a result, the concentration may not have been uniform throughout the sample. As a result, some of the absorbance values may be higher or lower, reducing the precision of the absorbance values.

- When preparing the saturated solutions, some of the mixtures may have been mixed more rigorously and as a result, the concentration of turmeric in some of the filtrates may have been higher or lower.

\section{Improvements and extensions:}

Now that I have outlined and analysed the strengths and weaknesses of the experiment, it is clear that the experiment needs to be improved or extended in order to ensure the accuracy, precision and validity of the data and the claims based on the data.

\section{Improvements:}

- If possible, the use of a reusable quartz cuvette may allow more precise absorbance measurements, however the limitation of this is that between each trial the cuvette would have to be washed and dried thoroughly, which would significantly increase the time needed for each trial

- Possibly testing each sample at different wavelengths and preparing calibration curves at each wavelength would permit more repetitions

- Using gloves when handling the cuvettes will ensure than no grease from one's fingers contaminates the results

- Mixing the samples within the cuvettes more thoroughly will ensure that the concentration of turmeric is uniform throughout the sample 


\section{Extensions:}

- Performing more repetitions, especially when preparing the calibration curves, will greatly increase the precision and reliability of the data

- Using a wider range of solvents will allow the hypothesis to be tested more rigorously, especially if an aprotic non-polar solvent (non-polar solvents that are unable to form hydrogen bonds) such as hexane is able to be tested, as none of the solvents tested were both aprotic and non-polar

There are two types of solubility, kinetic and thermodynamic. In this experiment, kinetic solubility was investigated but it may be worth investigating the thermodynamic solubility. This may be challenging, as curcumin degrades quickly in $\mathrm{NaOH}$ solutions.

\section{Acknowledgements}

I thank Mrs. Sivakumar for supporting me throughout both the research and writing processes. I also thank Glenunga International for providing various reagents, equipment and a laboratory space.

\section{References}

Brown, C. and Ford, M., 2014. Higher Level Chemistry. 2nd ed. Harlow: Pearson Education Limited.

Jagannathan, R., Abraham, P. and Poddar, P., 2012. Temperature-Dependent Spectroscopic Evidences of Curcumin in Aqueous Medium: A Mechanistic Study of Its Solubility and Stability. The Journal of Physical Chemistry B, [online] 116(50), pp.14533-14540. Available at: https://pubs.acs.org/doi/10.1021/jp3050516 [Accessed 6 June 2020].

Ncbi. 2010. Stabilization of Curcumin by Complexation with Divalent Cations in Glycerol/Water System.

[ONLINE] Available at: https://www.ncbi.nlm.nih.gov/pmc/articles/PMC2904446/. [Accessed 1 June 2020].

Ncbi. 2011. Turmeric, the Golden Spice. [ONLINE] Available at: https://www.ncbi.nlm.nih.gov/books/NBK92752/.

[Accessed 21 June 2020].

Priyadarsini, K., 2014. The Chemistry of Curcumin: From Extraction to Therapeutic Agent. Molecules, [Online]. 19, 0. Available at: https://www.researchgate.net/publication/269182694 The Chemistry of Curcumin From Extraction to Therapeutic Agent [Accessed 8 June 2020].

Science.cleapss.org.uk. 2019. Ethanol. [online] Available at: http://science.cleapss.org.uk/Resource/SSS060Ethanol.pdf [Accessed 18 June 2020].

Science.cleapss.org.uk. 2019. Hydrochloric acid. [online] Available at: http://science.cleapss.org.uk/Resource/SSS020-Hydrochloric-acid.pdf [Accessed 18 June 2020].

Science.cleapss.org.uk. 2019. Higher alcohols. [online] Available at: http://science.cleapss.org.uk/Resource/SSS066-Higher-alcohols-propanols-butanols-pentanols.pdf [Accessed 18 June 2020].

Science.cleapss.org.uk. 2019. Sodium hydroxide. [online] Available at: http://science.cleapss.org.uk/Resource/SSS031-Sodium-hydroxide.pdf Accessed 18 June 2020]. 
Science Direct. 2014. Colorimetry. [ONLINE] Available at: https://www.sciencedirect.com/topics/chemical-engineering/colorimetry\#: :text=Colorimetry\%20is\%20a \%20scientific\%20technique,is\%20proportional\%20to\%20the\%20absorbance.. [Accessed 21 June 2020].

Wikipedia. 2020. pH indicator. [ONLINE] Available at: https://en.wikipedia.org/wiki/PH indicator . [Accessed 21 June 2020]. 